\title{
Micro-thermography enables rapid inspection of defects in diode lasers
}

Anna Kozlowska, Jens W. Tomm, Piotr Wawrzyniak, and Fritz Weik

Micro-thermography, previously used for the inspection of semiconductor circuits, is now applied to detecting defects in diode lasers. A multi-channel measurement system is used to distinguish between pure thermal radiation and parasitic emissions. This allows effects related to both fast and slow degradation of diode lasers to be monitored, making micro-thermography a highly-attractive tool for device screening.

Diode lasers (DLs) are efficient light sources that are widely used in telecommunication, computer equipment, medicine, telemetry and material processing. Though the technology of lowpower devices has already reached a level of maturity, the reliability of high-power DLs remains an issue. Diode laser bars could be widely used-e.g. for optical pumping of solid-state lasers - but due to reliability problems they frequently lose when in competition even with conventional lamp sources. In order to increase reliability, manufacturers need a better way to inspect devices for defects before shipping the DLs to customers.

Past approaches used to identify the defects inside the diode laser include microscopic, spectroscopic, and electrical methods. ${ }^{1}$ These methods are helpful in understanding and solving the degradation problems, but their complexity prevents their use in an industrial environment. Micro-thermography seems to be an ideal solution, especially if we take into account the enormous progress in the field of infrared (IR) imaging: improved spatial resolution, quasi-real-time operation and high detection sensitivity. Thermography has already been implemented for the inspection of high-power laser bar stacks. ${ }^{2}$ Also, our recent work has been devoted to the analysis of various thermal problems in DLs and DL bars. ${ }^{3-5}$

Our basic idea for this measurement relies on the fact that both defect creation and accumulation are typically accompanied by a local temperature increase that can be directly detected by micro-thermography. Moreover, the extension of this measurement system to include two spectral channels, together with

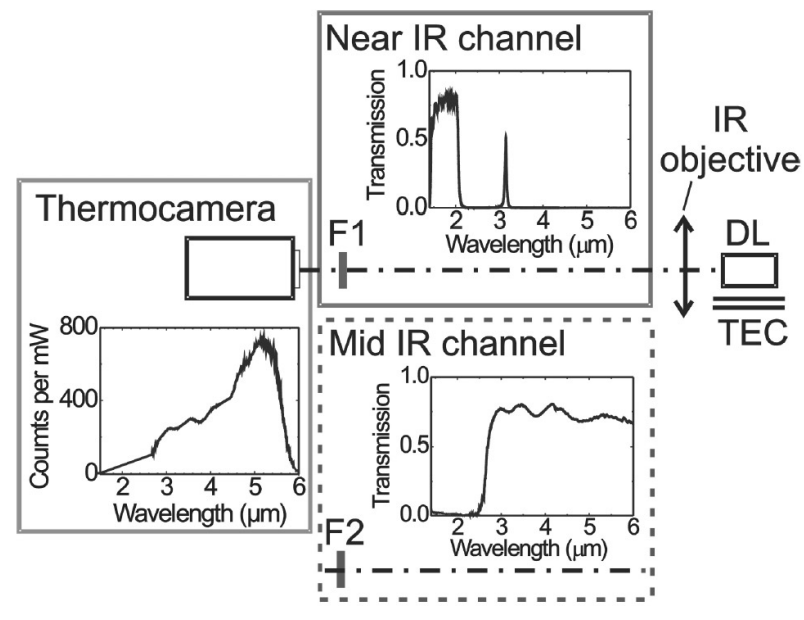

Figure 1. Multi-spectral thermocamera system inspects a diode laser $(D L)$ in two spectral ranges. Spectra show the sensitivity of the thermocamera as well as transmission curves of filters F1 and F2. TEC: thermoelectric cooler.

analysis of thermal transients, allows our system to distinguish between pure thermal radiation and any other parasitic emission, such as deep-level luminescence.

To investigate problems related to fast and slow degradation of diode lasers we used devices made in-house. These were based on a double-barrier separate confinement heterostructure emitting at $808 \mathrm{~nm} .^{6}$ Their design is intended to reduce fast-axis beam divergence. Figure 1 shows a schematic of our thermographic system. We used a camera sensitive in the wavelength range $1.5-5.5 \mu \mathrm{m}$. Two filters, F1 and F2, define spectral channels in the near and mid IR.

We captured mid-IR thermal images of DLs that were candidates for fast degradation. A DL with a hot spot at the front facet is shown in Figure 2(a). In the case of another DL, the defect is located deeper in the substrate and is visible as an ex- 

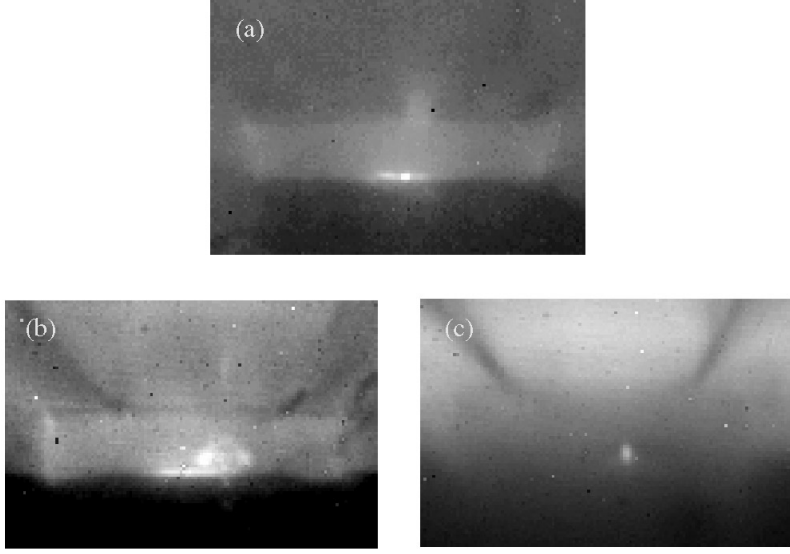

Figure 2. Thermographic images of defective diode lasers acquired in the mid IR. In (c), the focal point of the camera is shifted to $300 \mu \mathrm{m}$ below the laser's surface.
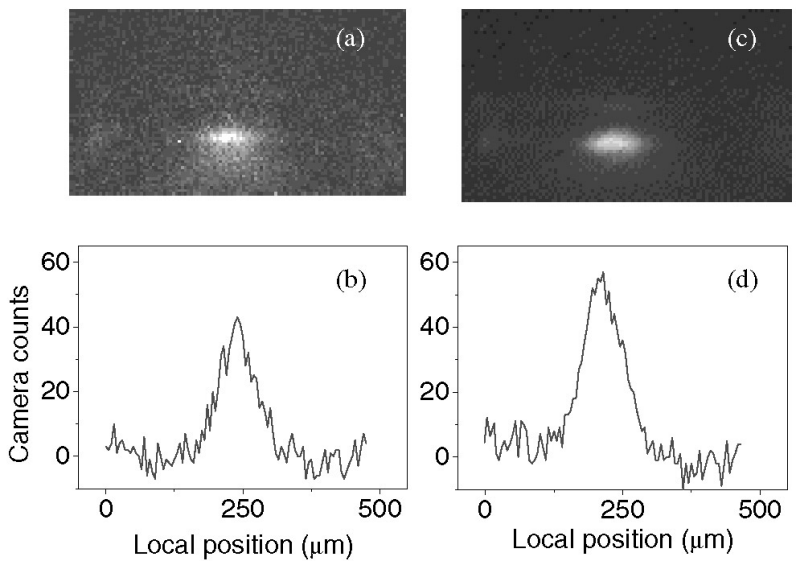

Figure 3. Front view thermographic images acquired in the near IR for a new (a) and aged diode laser (c) and their lateral profiles $(b)$ and (d), respectively, taken along the junction plane.

tended warmed area, see Figure 2(b). If we shift the focal plane from the facet to the defect inside the cavity, however, we can image a localized hot spot, see Figure 2(c). Moreover, we have demonstrated $^{3}$ that thermal profiling helps recognize defects that cannot be detected by simple visual inspection.

Near-IR images of diode lasers can show signs of slow degradation. We found that signals detected in this spectral channel are mainly caused by mid-gap deep-level luminescence. ${ }^{4,5} \mathrm{~A}$ comparison between a new DL and a DL aged for 3000 hours see Figure 3(a,b) and $(c, d)$ respectively - shows that, over time, the intensity of this type of luminescence increases in the near IR. Most probably, the concentration of deep-level defects increases during aging. On the other hand, re-absorption of light at the defect centers gives rise to increased heating, which was examined in the mid-IR range. ${ }^{5}$ The temperature increase as a result of long-term operation certainly limits the performance of the device.

Summarizing, we have used a micro-thermographic method to investigate effects responsible for both fast and slow degradation of diode lasers. The results presented here pave the way for an advanced reliability screening methodology for high-power diode lasers and laser bars.

\section{Author Information}

\section{Anna Kozlowska and Piotr Wawrzyniak \\ Laser Laboratory \\ Institute of Electronic Materials Technology \\ Warsaw, Poland \\ http://www.itme.edu.pl}

Dr Anna Kozlowska is the head of Laser Laboratory in the Institute of Electronic Material Technology in Warsaw (Poland). She is working on high-power diode laser technology and development of advanced characterization methods.

\section{Jens W. Tomm and Fritz Weik}

Max-Born-Institut fur Nichtlineare Optik und

${ }^{1}$ Kurzzeitspektroscopie

Berlin, Germany

http://www.mbi-berlin.de

\section{References}

1. M. Fukuda, Reliability and Degradation of Semiconductor Lasers and LEDs, Artech House, Boston, 1991.

2. B. L. Meadows, F. Amzajerdian, N. R. Baker, V. Sudesh, U. N. Singh, and M. J. Kavaya, Thermal characteristics of high-power, long pulse width, quasi-CW laser diode arrays, Proc. SPIE 5336, p. 203, 2004.

3. A. Kozlowska, J. W. Tomm, F. Weik, T. Elsaesser, M. Latoszek, M. Zbroszczyk M. Bugajski, B. Spellenberg, and M. Bassler, Analysis of thermal images from diode lasers: Temperature profiling and reliability screening, Appl. Phys. Lett. 86, p. 203503, 2005.

4. A. Kozlowska, P. Wawrzyniak, J. W. Tomm, F. Weik, and T. Elsaesser, Deep level emission from high-power diode laser bars detected by multi-spectral infrared imaging, Appl. Phys. Lett. 87, p. 153503, 2005.

5. A. Kozlowska, J. W. Tomm, P. Wawrzyniak, A. Malag, F. Weik, and M. Latoszek, Studies of the degradation mechanisms in high-power diode lasers using multi-channel micro-thermography, Proc. SPIE 5958, 2005.

6. A. Malag, Beam divergence and COD issues in double barrier separate confinement heterostrucure laser diodes, Bulletin of the Polish Academy of Sciences, Technical Sciences 53 (2), 2005. 